\title{
Efficiency of Competition in Insurance Markets with Adverse Selection
}

G. De Feo and J. Hindriks

Discussion Paper 2005-42

Département des Sciences Économiques de l'Université catholique de Louvain 


\title{
Efficiency of Competition in Insurance Markets with Adverse Selection
}

\author{
Giuseppe De Feo \\ CORE, Université Catholique de Louvain and University of \\ Napoli "Federico II" \\ Jean Hindriks \\ CORE, Université Catholique de Louvain
}

July 2005

\begin{abstract}
There is a general presumption that competition is a good thing. In this paper we show that competition in the insurance markets can be bad when there is adverse selection. Using the dual theory of choice under risk, we are able to fully characterize both the competitive and the monopoly market outcomes. When there are two types of risk, the monopoly dominates competition if and only if competition leads to market unravelling. When there are a continuum of types the efficiency of competition is less trivial. In effect monopoly is shown to provide better insurance but at the cost of driving out some agents from the market. Performing simulation for different distributions of risk, we find that monopoly in general performs (much) better than competition in terms of the realization of the gains from trade across all traders in equilibrium. The reason is that the monopolist can exploit its market power to relax the incentive constraints.
\end{abstract}

Keywords: monopoly, competition, non-expected utility, insurance, adverse selection.

JEL classification: G22, G82

We are grateful to Enrico Minelli, Francesca Stroffolini, Piero Tedeschi and Izabela Jelovac for helpful comments and suggestions. This work also benefitted from comments and feedback from seminar audiences at CORE and the Universities of Liege and Milano Bocconi. 


\section{Introduction}

In this paper we address the critical question: how and how well do competition on the markets handle the fundamental problems of information. With imperfect information, market actions or choices convey information and we know from earlier work (e.g. Rothschild and Stiglitz, 1976) that existence problems can arise in competitive markets because the slight change in the action of the informed side of the market discretely changes beliefs of the other side of the market. While information asymmetries inevitably arise, the extent to which they do so and their consequences on the realization of the gains from trade depend on the how the market is structured. This raises the fundamental question of the interplay between two forms of market imperfections: imperfect information and imperfect competition. There is no particular reason why competition should be better in the presence of imperfect information. The simplest way by which this would not be true is when the firm could exploit its market power to relax the incentive constraints.

The aim of this paper is to evaluate the efficiency of competition on the insurance market in the presence of adverse selection. Using the benchmark model of Rothschild and Stiglitz (1976), we contrast the competitive equilibrium outcome with the monopoly equilibrium outcome à la Stiglitz (1977) and we compare their relative efficiency. Following Rustichini et al (1994), the (expected) efficiency of an equilibrium is the fraction whose numerator is the expected gains from trade across all traders in the equilibrium and whose denominator is the expected gains from trade across all traders with full information. Using this criterion we compare the monopoly outcome with one seller of insurance contracts and many potential buyers with different risks against the competitive outcome imposing zero profit on each contract that might be offered in equilibrium. To deal with the possible non-existence of a competitive equilibrium à la Rothschild Stiglitz with a continuum of types (Riley, 2001) we refer to the concept of reactive equilibrium developed by Riley (1979) and Engers and Fernandez (1987) for which the Pareto-dominant full separating zero-profit outcome is the unique reactive equilibrium.

It is well known that models describing the effect of asymmetric information on the market performance can be distinguished according to how they model the market mechanism and how they define a market outcome. The conclusions then turn out to be very sensitive to minor modelling changes. To handle this difficulty we choose not to model a particular market mechanism but rather take the fundamental feature of all market mechanisms, voluntary participation in trade and the incentive constraints, and apply the revelation principle to study the relative efficiency of competition. 
Our paper continues a line of research begun by Stiglitz (1977), who analyzed monopoly insurance mostly with two types of agent with expected utility, and compared the equilibrium outcome with the (twotype) competitive outcome. However his comparison analysis did not come out neatly when dealing with a continuum of types. Dalhby (1987) studies the same issue in a two-type insurance model with expected utility, but again without using the expected efficiency criterion to compare competition and monopoly. We perform this analysis in a non-expected utility approach and with a continuum of risks. It turns out that by using the dual theory approach of Yaari (1987), we are able to provide a clearcut comparison between monopoly and competition.

The dual theory has the property that utility is linear in income, and risk aversion is expressed entirely by a transformation of probabilities in which bad outcomes are given relatively higher weights and good outcomes are given relatively lower weights. In our simple two-state model the probability of bad outcome is weighted up by a loading factor. As we shall see this formulation of risk aversion without diminishing marginal utility allows the derivation of a rich set of insights. Although most of the classical results in insurance theory appear to be robust to such departures from the expected utility model, one important implication to the demand of insurance deserves to be emphasized and will play a major role in the rest of the analysis: under the dual theory, a risk averse individual has constant marginal willingness to pay for insurance whereas under expected utility a risk averse individual has decreasing marginal willingness to pay. ${ }^{1}$ A related key difference is about the order of risk aversion. ${ }^{2}$ When risk aversion is of order 1 as in the DT, it could be optimal for a policyholder to buy full insurance even above the fair price. This is because he derives positive benefits from the last dollar of coverage. By contrast under EU, risk aversion is of the second order and the benefit from the last dollar of coverage is zero. Therefore nobody would find profitable to buy complete insurance slightly above fair price in the EU model eventhough this is common practice. ${ }^{3}$

Recently some works by Jeleva and Villeneuve (2004) and Chassagnon and Villeneuve (2005) deal with a seemingly related argument: the insurance market under adverse selection with subjective risk perception. In these works, risk aversion is still expressed by the decreasing

\footnotetext{
${ }^{1}$ Fortunately, as shown in Doherty and Eeckhoudt (1995) and Machina (1989, 1995) many of the implications of expected utility theory for the optimal insurance design carry over to the non-expected utility approach.

${ }^{2}$ For the definition of the order of risk aversion, see Segal and Spivak (1990). An excellent survey of the various definitions of risk aversion is provided in Cohen (1995).

${ }^{3}$ See Mossin (1968).
} 
marginal utility of wealth, but probabilities are modified in the expected utility function according to a subjective perception. In the monopolist framework with two types a rich set of equilibrium allocation is defined, depending on the relative perception of the risk of the two types. ${ }^{4}$ Among these results, a pooling equilibrium is a possible outcome, just as in our paper, essentially for the same reason: the order of risk aversion. The second best allocation with two types differs from our results (see Section $3){ }^{5}$ Indeed, by allowing for overinsurance and restricting the analysis to utility function with smooth indifference curves ("no kink" at full coverage) they show that there is no positive profits in the second best allocation, and that there is no pooling contract with full coverage in the second best. These conclusions rest heavily on the fact that the no-kink assumption implies that "pessimistic types", that have a perception of the risk higher than reality, prefer risky outcomes (with over-insurance) to sure outcome (with full insurance). Moreover this approach is not appropriate for our welfare analysis. The fact that agents make mistake in their perception of the risk makes the welfare analysis of competition potentially misleading (i.e. should we take into account such misperception of risk when comparing competitive outcome versus the monopoly outcome?).

It would be absurd to suggest that the dual theory provides a better model than the expected utility. The latter has obvious appeal and has provided so many useful results in insurance theory. Nonetheless, we feel there is some gain from studying the properties of our simple non-expected utility model, even if only to derive some clear insights on the efficiency of competition in the presence of adverse selection. Indeed another distinctive property of insurance under DT is that the demand of insurance cannot decrease with wealth. In contrast the EU model makes the comparison between competition and monopoly difficult since by charging a higher premium (relative to competition) for a given coverage the monopoly increases the marginal willingness to pay for insurance. ${ }^{6}$ As a result Dahlby (1987) showed that equilibrium coverage can be either higher or lower in a monopoly.

The key finding is that the monopoly outcome, in general, is more efficient than the competitive outcome (according to our expected efficiency criterion). The reason why monopoly performs better than competition is that the monopolists can exploit its market power to offer contracts that better satisfy the incentive constraints. More precisely, the monopo-

\footnotetext{
${ }^{4}$ Jeleva and Villeneuve (2004).

${ }^{5}$ Chassagnon and Villeneuve (2005).

${ }^{6}$ Dahlby (1987) shows that the marginal willingness to pay for insurance is a decreasing function of wealth when the absolute risk aversion is decreasing.
} 
list can offer contracts with implicit transfers across agent types that can relax the incentive constraints and implement a larger set of allocations. This is one of many examples of the interplay between market imperfections (see Stiglitz 1975 and Jaffee and Stiglitz 1990). The economy, in effect has to trade off between two different imperfections: imperfections of information or imperfections of competition, with no particular reason that these imperfections will be balanced optimally.

Our main finding about the inefficiency of competition has to be contrasted with recent work on the (asymptotic) efficiency of competition based on the idea that asymmetry in agent's information is relatively unimportant in a large economy (obtained by a replication process) because any single agent has only a small amount of information not known by the other agents. ${ }^{7}$ This is the notion of informational smallness. As Gul and Postlewaite noted, this result holds in private value information problems in which agents have private information that is of direct relevance only to themselves (i.e. the agents' utility functions depend only on their own type). This is obviously not the case for the insurance problem (and for the adverse selection problem in general). Indeed in our insurance problem with a continuum of types it might seem that each agent is informationally small and yet the market outcome is very far from the full information outcome. The reason is that each agent remains informationally large in this context. Muthoo and Mutuswami (2005) obtain a similar result for the "lemons" markets. They show that efficiency is decreasing in the degree of market competition (measured by the number of sellers, fixing the number of buyers). The driving force for their result is different however. They show that first-best surplus is increasing in the number of sellers (of either high or low quality good) but the realized market surplus is unchanged. The reason is that, in the first-best, the probability of trade with a high quality seller increases with the number of sellers. In contrast the incentive constraints imply that the probability with which a high-quality seller will trade cannot be greater than the probability with which a low quality seller will trade, which is independent of the number of sellers.

The paper is organized as follows. In Section 2 we present the model. Section 3 contains the full information equilibrium and efficiency analysis of competitive and monopoly markets in the simple two-type case. In Section 4 the analysis is extended to the continuum of types and simulation results are provided about the expected efficiency of competitive and monopoly markets. Section 5 concludes.

\footnotetext{
${ }^{7}$ See Gul and Postlewaite (1992), Gresik Satterthwaite (1989), Satterthwaite and Williams (1989), Rustichini et al (1994).
} 


\section{The model}

There are two possible states of the world: the "no accident" state and the "accident" state. Individuals differ only by their probability of accident, in which case they face a (fixed) damage $d=1$. There is no moral hazard since individuals cannot affect their probability of accident which is fixed. There is a continuum of risk in the economy distributed according to a cumulative probability function $F(\theta)$ with density $f(\theta)>0$ on a closed and compact interval $\theta \in[\underline{\theta}, \bar{\theta}]$ (with $0 \leq \underline{\theta}<\bar{\theta}<1$ ).

Adverse selection is introduced by assuming that individual risk is private information, while the distribution of risks is common knowledge. We model individuals' risk preferences using Yaari (1987)'s dual theory (DT). We first give a general description of this approach before applying it to our model. Let wealth $X$ be a random variable distributed over $[\underline{x}, \bar{x}]$ according to the distribution function $\Psi(x)$. Yaari's representation of preferences is dual to the expected utility theory (EU) in the sense that it is linear in wealth but non linear in probabilities. Probabilities are transformed by a function $\Phi$ defined on the distribution function $\Psi(x) .{ }^{8}$ More precisely, DT preferences over $X$ are given by

$$
V(X)=\int x \Phi^{\prime}(\Psi(x)) d \Psi(x)
$$

where $\Phi(0)=0, \Phi(1)=1$ and $\Phi^{\prime}()>.0 . \Phi^{\prime}($.$) are non-negative weights$ adding up to one. Attitude towards risk is conveyed entirely by the shape of $\Phi($.$) . Risk aversion is characterized by the concavity of \Phi($.$) ,$ i.e. $\Phi^{\prime \prime}()<$.0 . In this case, bad outcomes (with low $\Psi(X)$ ) receive higher weights than good outcomes (with high $\Psi(X)$ ). In other words, $V(X)$ is the certainty-equivalent of $X$ computed as a weighted average of outcomes in which bad outcomes are given high weight while good outcomes are given low weight. Since $V(X)$ is linear in wealth, this approach separates attitude towards risk from attitude towards wealth.

We now apply DT to our simple two-state setting. For an individual with wealth $w$ facing a damage $d=1$ with probability $\theta$, insurance contract with premium $\pi>0$ and coverage rate $\delta \in[0,1]$ yields the random variable $X=(w-\pi-(1-\delta), \theta ; w-\pi, 1-\theta)$. We thus define the utility associated to this insurance contract as

$$
\begin{aligned}
V(\pi, \delta ; \theta) & =\phi(\theta)(w-\pi-(1-\delta))+(1-\phi(\theta))(w-\pi) \\
& =w-\pi-\phi(\theta)(1-\delta)
\end{aligned}
$$

\footnotetext{
${ }^{8}$ Alternatively this probability transformation function could be defined on the decumulative distribution function $1-\Psi$ such as in Yaari (1987).
} 
where risk aversion is represented by $\phi(\theta)>\theta($ and $1-\phi(\theta)<1-\theta) .{ }^{9}$ In this paper, we further assume that $\phi(\theta)=(1+\alpha) \theta$, with $0 \leq \alpha \leq \frac{1-\bar{\theta}}{\bar{\theta}}$ (the upper bound guaranteeing that $\phi(\theta) \leq 1 \forall \theta$ ). Making $\alpha$ independent of $w$ accords with our desire to disentangle risk aversion from income and will greatly simplify the analysis. Using this formulation, type- $\theta$ utility function from insurance contract $(\pi, \delta)$ is

$$
V(\pi, \delta ; \theta)=\omega-\pi-(1+\alpha)(1-\delta) \theta
$$

where the utility loss from the residual risk $(1-\delta) \theta$ is inflated by the markup factor $1+\alpha$. Now, comparing the utility with insurance against the utility without insurance we can define the reservation premium for each type. This is the premium $\pi \equiv \tilde{\pi}(\theta)$ that solves

$$
\begin{aligned}
V(\pi, \delta ; \theta) & =V(0,0 ; \theta) \\
\omega-\pi-(1+\alpha)(1-\delta) \theta & =\omega-(1+\alpha) \theta
\end{aligned}
$$

so that the reservation premium of type $\theta$ for coverage $\delta$ is:

$$
\tilde{\pi}(\delta ; \theta)=(1+\alpha) \delta \theta
$$

Moreover the surplus of the agent is defined as the difference between the reservation price and the price effectively paid:

$$
\begin{aligned}
S(\pi, \delta ; \theta) & =\tilde{\pi}(\delta ; \theta)-\pi \\
& =(1+\alpha) \delta \theta-\pi
\end{aligned}
$$

Assuming $\pi>0$, with free participation, those agents receiving $\delta<0$ will drop out of the market.

It is straightforward to see that the functions $V$ and $S$ have the Single-Crossing property in the contract space- $(\pi, \delta)$, because the marginal value of coverage is increasing in $\theta$. This property implies the Increasing Difference (ID) property.

A function $f: \Delta \times \Theta \rightarrow \mathbb{R}$, where $\Delta, \Theta \subset \mathbb{R}$, has Increasing Differences if the difference $f\left(\delta^{\prime \prime}, \theta\right)-f\left(\delta^{\prime}, \theta\right)$ is increasing in $\theta \in \Theta \forall \delta^{\prime \prime}, \delta^{\prime}$ $\in \Delta$ such that $\delta^{\prime \prime}>\delta^{\prime}$.

\footnotetext{
${ }^{9}$ Note that in our model with a discrete random variable, risk aversion translates into the transformation of the discrete density function $\phi(\theta)>\theta$ rather than the concave transformation of the distribution function $\Phi^{\prime \prime}(\Psi)<0$ as for continuous random variable. In both cases risk aversion implies that bad outcomes are given higher weight and good outcomes lower weight.
} 
The ID property of $V$ and $S$ is due to the fact that these functions are continuously differentiable on a closed interval $\delta \in[0,1]$ with the cross-derivatives

$$
\frac{\partial^{2} S}{\partial \delta \partial \theta}=\frac{\partial^{2} V}{\partial \delta \partial \theta}=(1+\alpha)>0
$$

As a prelude to our analysis we will first study the two-type case. This will provide the preliminary insights on the efficiency of competition in the insurance market.

\section{The two-type case}

There are two types of risk. A proportion $p$ has risk $\underline{\theta}$ and a proportion $(1-p)$ has risk $\bar{\theta}$. We derive first, as a benchmark, the first-best allocations. Then we compare this outcome with the competitive and monopoly insurance outcomes.

\subsection{First-best}

The first best solution, when there is no problem of information about the type of the agent, is easily derived by maximizing the expected surplus across all traders subject to the participation constraints. The optimization programme is:

$$
\max _{\substack{\pi(.) \geq 0 \\ \delta(.) \in[0,1]}}(1-p) S(\pi(\bar{\theta}), \delta(\bar{\theta}) ; \bar{\theta})+p S(\pi(\underline{\theta}), \delta(\underline{\theta}) ; \underline{\theta})+\Pi
$$

subject to

$$
\begin{aligned}
&(1-p)[\pi(\bar{\theta})-\delta(\bar{\theta}) \bar{\theta}]+ p[\pi(\underline{\theta})-\delta(\underline{\theta}) \underline{\theta}] \equiv \Pi \geq 0 \\
& S(\pi(\underline{\theta}), \delta(\underline{\theta}) ; \underline{\theta}) \geq 0 \\
& S(\pi(\bar{\theta}), \delta(\bar{\theta}) ; \bar{\theta}) \geq 0
\end{aligned}
$$

Substituting for the surplus functions and the profit functions, the programme reduces to:

$$
\max _{\substack{\pi(.) \geq 0 \\ \delta(.) \in[0,1]}}(1-p) \alpha \delta(\bar{\theta}) \bar{\theta}+p \alpha \delta(\underline{\theta}) \underline{\theta}
$$

subject to

$$
\begin{aligned}
(1-p)[\pi(\bar{\theta})-\delta(\bar{\theta}) \bar{\theta}]+p[\pi(\underline{\theta})-\delta(\underline{\theta}) \underline{\theta}] \geq 0 \\
(1+\alpha) \underline{\theta} \delta(\underline{\theta})-\pi(\underline{\theta}) \geq 0 \\
(1+\alpha) \bar{\theta} \delta(\bar{\theta})-\pi(\bar{\theta}) \geq 0
\end{aligned}
$$


The solution is:

$$
\begin{aligned}
\delta(\bar{\theta}) & =\delta(\underline{\theta})=1 \\
\pi(\bar{\theta}) & \in[\bar{\theta},(1+\alpha) \bar{\theta}] \\
\pi(\underline{\theta}) & \in[\underline{\theta},(1+\alpha) \underline{\theta}]
\end{aligned}
$$

All the agents are provided with full insurance and the premium charged defines the way the surplus is split among firms and agents. The firms get all the surplus when the premia are set equal to the reservation prices $\pi(\underline{\theta})=(1+\alpha) \underline{\theta}$ and $\pi(\bar{\theta})=(1+\alpha) \bar{\theta}$, while the agents get all the surplus when the premia are set equal to the fair prices $\pi(\underline{\theta})=\underline{\theta}$ and $\pi(\bar{\theta})=\bar{\theta}$.

Total surplus in the first-best is then

$$
W^{F B}=(1-p) \alpha \bar{\theta}+p \alpha \underline{\theta}
$$

\subsection{Second-best}

The set of second-best allocation (also called constrained Pareto optimum) is characterized by the set of allocations satisfying the Incentive Compatibility constraints together with the participation constraints. ${ }^{10}$ It solves the following programme:

$$
\max _{\substack{\pi(.) \geq 0 \\ \delta(.) \in[0,1]}}(1-p) \alpha \delta(\bar{\theta}) \bar{\theta}+p \alpha \delta(\underline{\theta}) \underline{\theta}
$$

subject to

$$
\begin{aligned}
(1-p)[\pi(\bar{\theta})-\delta(\bar{\theta}) \bar{\theta}]+p[\pi(\underline{\theta})-\delta(\underline{\theta}) \underline{\theta}] \geq 0 \\
(1+\alpha) \underline{\theta} \delta(\underline{\theta})-\pi(\underline{\theta}) \geq 0 \\
(1+\alpha) \bar{\theta} \delta(\bar{\theta})-\pi(\bar{\theta}) \geq 0 \\
(1+\alpha) \underline{\theta} \delta(\underline{\theta})-\pi(\underline{\theta}) \geq(1+\alpha) \underline{\theta} \delta(\bar{\theta})-\pi(\bar{\theta}) \\
(1+\alpha) \bar{\theta} \delta(\bar{\theta})-\pi(\bar{\theta}) \geq(1+\alpha) \bar{\theta} \delta(\underline{\theta})-\pi(\underline{\theta})
\end{aligned}
$$

The participation constraint of high type (6) is implied by (5) and (8) and can be disregarded. Also following standard argument of no-distortionat-the-top, the surplus is maximized for $\delta(\bar{\theta})=1$. The incentive constraint of the low type (7) is typically not binding. Using these facts the optimization programme reduces to

$$
\max _{\substack{\pi(\underline{\theta}), \pi(\bar{\theta}) \geq 0 \\ \delta(\underline{\theta}) \in[0,1]}}(1-p) \alpha \bar{\theta}+p \alpha \delta(\underline{\theta}) \underline{\theta}
$$

\footnotetext{
${ }^{10}$ This is second-best efficiency in the sense of Harris and Townsend (1981) according to which an allocation is second-best efficient if it is Pareto optimal within the set of allocations that are feasible under the incentive constraints and the participation constraints.
} 
subject to

$$
\begin{aligned}
(1-p)[\pi(\bar{\theta})-\bar{\theta}]+p[\pi(\underline{\theta})-\delta(\underline{\theta}) \underline{\theta}] & \geq 0 \\
(1+\alpha) \underline{\theta} \delta(\underline{\theta})-\pi(\underline{\theta}) & \geq 0 \\
(1+\alpha) \bar{\theta}-\pi(\bar{\theta}) & \geq(1+\alpha) \bar{\theta} \delta(\underline{\theta})-\pi(\underline{\theta})
\end{aligned}
$$

The solution to this optimization problem is given in the following proposition.

Proposition 1 There exists $p^{*}<1$ such that,

- for $p<p^{*}$ the second-best outcome is a separating contract with

$$
\begin{aligned}
& \delta(\underline{\theta})<\delta(\bar{\theta})=1 \\
& \pi(\underline{\theta})=(1+\alpha) \underline{\theta} \delta(\underline{\theta})<\pi(\bar{\theta})=\pi(\underline{\theta})+(1+\alpha)(1-\delta(\underline{\theta})) \bar{\theta}
\end{aligned}
$$

- for $p \geq p^{*}$ the second-best outcome is a pooling contract with

$$
\begin{aligned}
\delta(\underline{\theta}) & =\delta(\bar{\theta})=1 \\
\pi(\underline{\theta}) & =\pi(\bar{\theta}) \in[(1-p) \bar{\theta}+p \underline{\theta},(1+\alpha) \underline{\theta}]
\end{aligned}
$$

Proof. See Appendix.

Figure 1 depicts the first-best and second-best expected surplus as a function of $p$, no matter how the surplus is split between agents and firms. The Second Best coincides with the First Best when the proportion of low risks is sufficiently high to sustain a pooling outcome.

\subsection{Monopoly}

The monopoly maximizes profit subject to the IC and IR constraints of the two types:

$$
\max _{\substack{\pi() \geq 0 \\ \delta() \in[0,1]}} p[\pi(\underline{\theta})-\delta(\underline{\theta}) \underline{\theta}]+(1-p)[\pi(\bar{\theta})-\delta(\bar{\theta}) \bar{\theta}]
$$

subject to (5),(6),(7) and (8). Standard result in monopolist screening problem implies that the Individual Rationality constraint of the low type, (5), and the Incentive Compatibility constraint of the high type, (8), are binding. So the IC for the low type (7) is not binding. Moreover, as in the second-best outcome, the monopoly profit is maximized for $\delta(\bar{\theta})=1$. The monopoly outcome is the following.

Proposition 2 In a monopoly insurance market, there exists $p^{\circ}<1$ (with $p^{\circ}>p^{*}$ ) such that, 


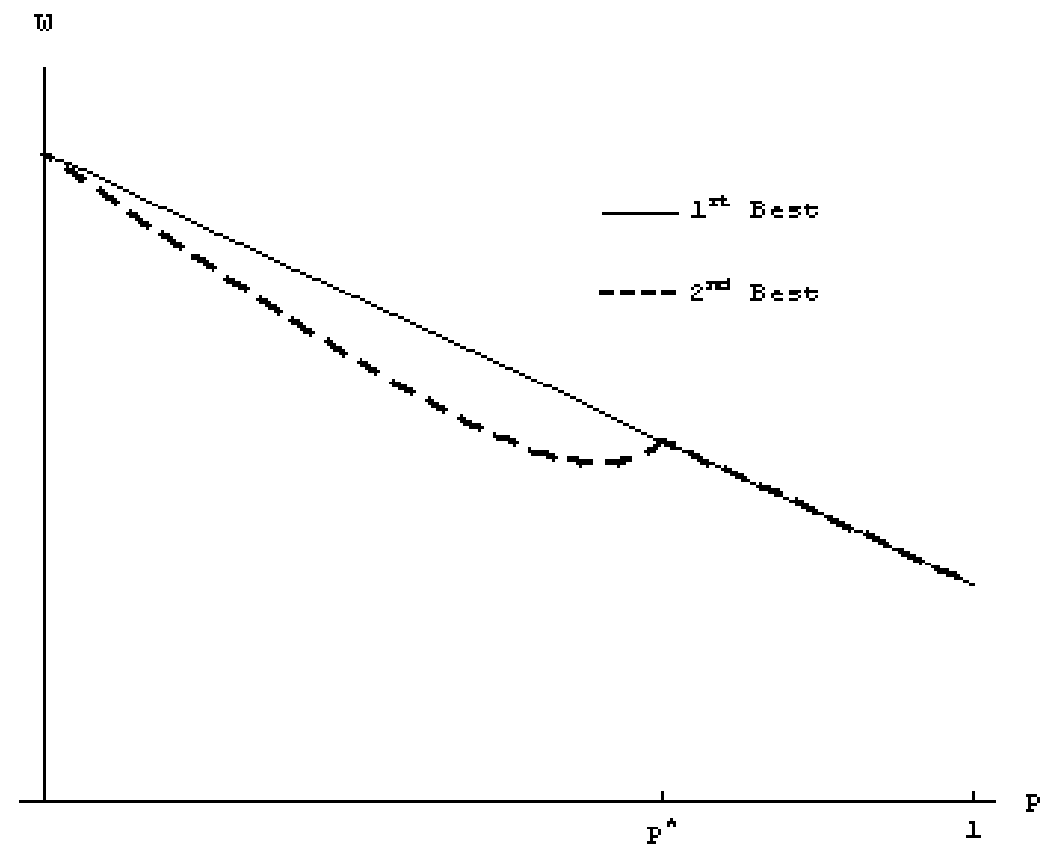

Figure 1: Comparison of the First and Second Best (as a function of $p$ ).

- for $p<p^{\circ}$ the monopoly outcome is a separating contract with

$$
\begin{aligned}
& \delta(\underline{\theta})=0, \delta(\bar{\theta})=1 \\
& \pi(\underline{\theta})=0, \pi(\bar{\theta})=(1+\alpha) \bar{\theta}
\end{aligned}
$$

- for $p \geq p^{\circ}$ the monopoly outcome is a pooling contract with

$$
\begin{aligned}
\delta(\underline{\theta}) & =\delta(\bar{\theta})=1 \\
\pi(\underline{\theta}) & =\pi(\bar{\theta})=(1+\alpha) \underline{\theta}
\end{aligned}
$$

Proof. see Appendix

The interpretation of the threshold $p^{\circ}$ is clear. Consider increasing the insurance coverage offered to the low type by $\Delta \delta(\underline{\theta})$. The net revenue from this type rises by an amount $\alpha \underline{\theta} \Delta \delta(\underline{\theta})$. This reduces revenue from the high-type to maintain the schedule incentive compatible. Since the indifference curves are linear, this revenue loss is just equal to the difference in the slopes of the indifference curves of the two types times the change in the coverage rate: $(1+\alpha)(\bar{\theta}-\underline{\theta}) \Delta \delta(\underline{\theta})$. Weighting these costs and benefits of increasing $\delta(\underline{\theta})$ by the proportion of each type, we have that it is (weakly) profitable if and only if $p \alpha \underline{\theta}-(1-p)(1+\alpha)(\bar{\theta}-\underline{\theta}) \geq 0$. 
The existence of a pooling monopoly equilibrium is in sharp contrast to the expected utility result. Indeed, Stiglitz (1977) showed that pooling equilibria are always dominated by separating equilibria where the lowrisk individuals obtain partial insurance and do not obtain any surplus. The reason is that expected utility implies that the marginal value of insurance is equal to the fair price at the full coverage point (i.e., SecondOrder risk aversion). So, starting from a pooling equilibrium with full coverage, a slight reduction in the coverage with offsetting reduction in premium to maintain the low type indifferent, does not affect the profit earned on that type, but it relaxes the incentive constraint on the high risk and allows the monopolist to extract more of the surplus from the high risk. With non-expected utility this argument does not apply because we have a First Order risk aversion. This implies that the marginal value of insurance is greater than the fair price at the full coverage point. Because the indifference curve of the low type is steeper than the fair price, a reduction of the coverage on the low types with offsetting reduction of the premium to maintain this type indifferent, will produce a first-order reduction in profits. If the proportion of low type is high enough the pooling equilibrium is stable.

\subsection{Comparison with competition}

In the competitive insurance market, contracts make zero profit. Following Rothschild and Stiglitz (1976) there exists no pooling equilibrium. This precludes any cross-subsidization between types as in the monopoly case. In fact, the competitive equilibrium can be represented by the following programme in which the surplus for each type is maximized subject to the IC and IR constraints plus the zero-profit conditions on each contract, that is:

$$
\max _{\substack{\pi(.) \geq 0 \\ \delta(.) \in[0,1]}} S(\pi(\theta), \delta(\theta) ; \theta) \quad \forall \theta=\{\underline{\theta}, \bar{\theta}\}
$$

subject to $(5),(6),(7),(8)$ and

$$
\begin{array}{r}
\pi(\underline{\theta})-\delta(\underline{\theta}) \underline{\theta}=0 \\
\pi(\bar{\theta})-\delta(\bar{\theta}) \bar{\theta}=0
\end{array}
$$

A separating equilibrium exists depending on the distribution of types. In the separating equilibrium, zero-profit conditions (12) and (13) imply fair price on each contract, so that the participation constraints (5) and (6) are not binding and only the Incentive Compatibility constraint (8) is binding,

$$
(1+\alpha) \bar{\theta} \delta(\bar{\theta})-\pi(\bar{\theta})=(1+\alpha) \bar{\theta} \delta(\underline{\theta})-\pi(\underline{\theta})
$$


where by (12) and (13)

$$
\begin{aligned}
\pi(\bar{\theta}) & =\delta(\bar{\theta}) \bar{\theta} \\
\pi(\underline{\theta}) & =\delta(\underline{\theta}) \underline{\theta}
\end{aligned}
$$

Using the standard no-distortion-at-the-top argument $\delta(\bar{\theta})=1$, and substituting back this expression and the fair prices into the incentive constraint gives the equilibrium coverage rate for the low type,

$$
\delta(\underline{\theta})=\frac{\alpha \bar{\theta}}{(\bar{\theta}-\underline{\theta})+\alpha \bar{\theta}}
$$

The low-type weakly prefers this separating contract to the pooling contract (with full insurance) if

$$
(1+\alpha) \underline{\theta}-(p \underline{\theta}+(1-p) \bar{\theta}) \leq \alpha \delta(\underline{\theta}) \underline{\theta}
$$

Substituting for $\delta(\underline{\theta})$, we get

$$
p \leq \frac{(1+\alpha)(\bar{\theta}-\underline{\theta})}{(1+\alpha)(\bar{\theta}-\underline{\theta})+\alpha \underline{\theta}}=p^{\circ}
$$

It is immediately verified that welfare is higher with competition when this condition is satisfied. High-risk individuals obtain full coverage at fair price (which is the full information allocation) and low-risk individuals obtain partial coverage, whereas under monopoly the low-risk individuals are not able to buy insurance. If this condition does not hold, the competitive market unravels and the monopoly dominates with the low-risk individuals being now able to buy full insurance. Thus we have the following proposition,

Proposition 3 There exists $p^{\circ}<1$ (with $p^{\circ}>p^{*}$ ) such that,

- for $p \leq p^{\circ}$ the surplus is higher under competition

- for $p>p^{\circ}$ the surplus is lower under competition.

Figure 2 depicts the competition and the monopoly surplus relative to the second-best allocation.

The bottom line is that, with two-type, monopoly insurance can dominate competition only if insurance market unravels under competition (i.e. $p>p^{\circ}$ ). In this case monopoly can in fact provide full insurance to both types. When the proportion of low type is sufficiently small, $p<p^{\circ}$, it pays the monopolist to exclude the low-risk individuals, and competition dominates by supplying insurance to both low-risk and high-risk individuals (although different contracts). 


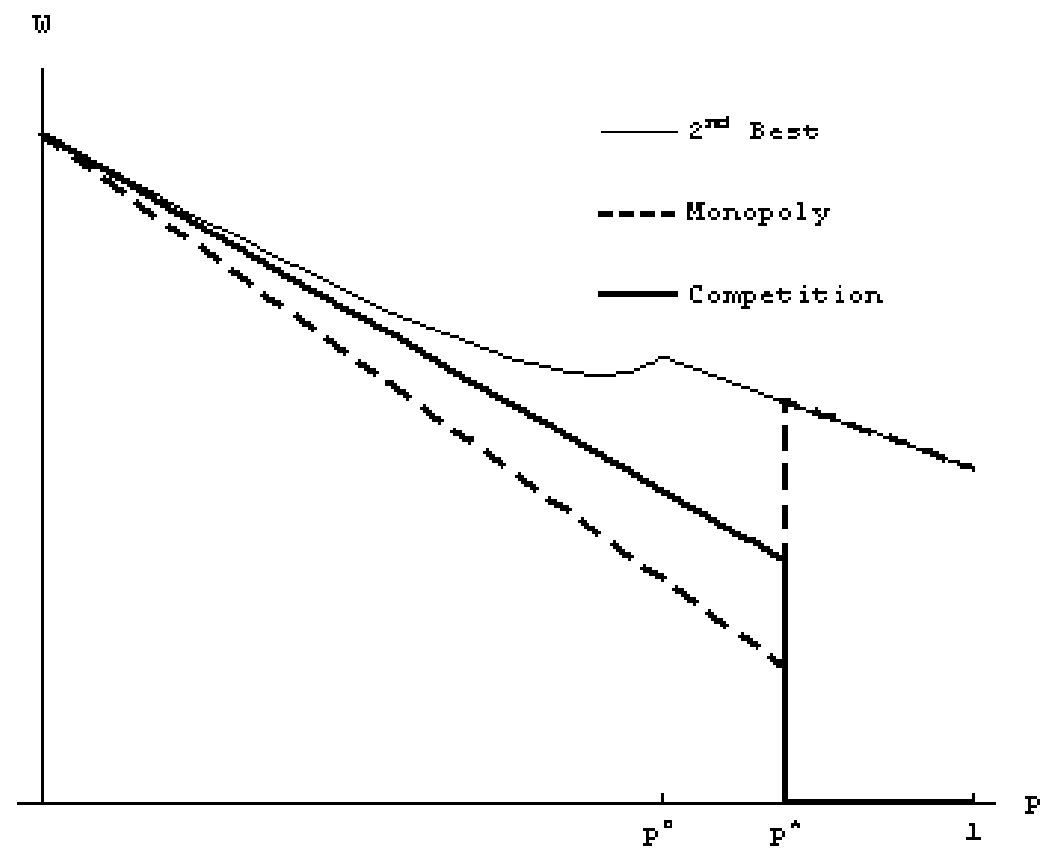

Figure 2: Comparison of the monopolist equilibria with the competitive equilibria and the second best (as a function of $p$ ).

Before proceeding to the analysis of the continuum case, we ask why the threshold $p^{\circ}$ is the same with competition and the monopoly. As argued earlier, with monopoly the threshold $p=p^{\circ}$ equates the expected revenue gain of increasing coverage to the low-risk individuals by $\Delta \delta(\underline{\theta})$; that is $p \alpha \underline{\theta} \Delta \delta(\underline{\theta})$ against the expected revenue loss on the high-risk individuals to keep the schedule incentive compatible, which by linearity is just equal to the difference in the slopes of the indifference curves of the two types times the change in the the coverage rate: $(1-p)(1+\alpha)(\bar{\theta}-\underline{\theta}) \Delta \delta(\underline{\theta})$.

With competition the zero-profit condition must hold and the threshold for a separating equilibrium $p=p^{\circ}$ is such that the low-risk individuals are indifferent between the separating contract with partial coverage and a zero-profit pooling contract with full coverage. From the indifference condition, all the rent that the low-risk-individuals make with the increased coverage of the pooling contract must be fully extracted, so the revenue gain is $p \alpha \underline{\theta} \Delta \delta(\underline{\theta})$ which, in order to maintain zero-profit, has to be equal to the revenue loss on the high-risk individuals $(1-p)(1+\alpha)(\bar{\theta}-\underline{\theta}) \Delta \delta(\underline{\theta})$. Thus the condition is the same as for the monopoly. 


\section{Continuum case}

\subsection{Monopoly}

In this section we study the equilibrium outcome of monopoly insurance with a continuum of risks. The optimization problem of the monopolist is:

$$
\max _{\pi(\theta), \delta(\theta)} \int_{\underline{\theta}}^{\bar{\theta}}[\pi(\theta)-\delta(\theta) \theta] d F(\theta)
$$

subject to

$$
\begin{array}{ll}
V(\pi(\theta), \delta(\theta) ; \theta) \geq V(0,0 ; \theta) & \forall \theta \in[\underline{\theta}, \bar{\theta}] \\
V(\pi(\theta), \delta(\theta) ; \theta) \geq V(\pi(\hat{\theta}), \delta(\hat{\theta}) ; \theta) & \forall \theta, \hat{\theta} \in[\underline{\theta}, \bar{\theta}]
\end{array}
$$

where (14) is the set of participation constraints and (15) denotes the set of incentive constraints. Analyzing the set (14) we can see that

$$
V(\pi(\underline{\theta}), \delta(\underline{\theta}) ; \underline{\theta}) \geq V(0,0 ; \underline{\theta})
$$

must be binding, for otherwise it would be possible to increase $\pi(\theta)$ $\forall \theta>\underline{\theta}$. This is the classical monopoly result of full rent extraction at the bottom. ${ }^{11}$ As a result all the agents with $\theta>\underline{\theta}$ are left with information rent, and so their participation constraints are not binding.

In the following Proposition the monopolist outcome is summarized.

Proposition 4 In a monopoly insurance market with a continuum of risk, there exists

$$
\theta^{*}=\frac{1+\alpha}{\alpha h\left(\theta^{*}\right)}
$$

with $h($.$) the non-decreasing hazard rate function, such that,$

- for $\theta<\theta^{*}$ the equilibrium contract is

$$
\begin{aligned}
& \delta(\theta)=0 \\
& \pi(\theta)=0
\end{aligned}
$$

- for $\theta \geq \theta^{*}$ the equilibrium contract is

$$
\begin{aligned}
\delta(\theta) & =1 \\
\pi(\theta) & =(1+\alpha) \theta^{*}
\end{aligned}
$$

\footnotetext{
${ }^{11}$ See for example Laffont and Tirole (1993).
} 
Proof. see Appendix

Therefore the solution is characterized by a (pooling) contract that offers full coverage to all $\theta \geq \theta^{*}$ with a premium extracting all the surplus from type $\theta^{*}$ and no insurance to all $\theta<\theta^{*}$. The equilibrium payoff of type $\theta$ under monopoly is:

$$
\begin{array}{ll}
V^{m}(\theta)=\omega-(1+\alpha) \theta^{*} & \forall \theta \geq \theta^{*} \\
V^{m}(\theta)=\omega-(1+\alpha) \theta & \forall \theta<\theta^{*}
\end{array}
$$

Rewriting $h\left(\theta^{*}\right)=\frac{f(\theta)}{1-F(\theta)}$ the pivotal type solves

$$
\alpha \theta^{*} f\left(\theta^{*}\right)=(1+\alpha)\left(1-F\left(\theta^{*}\right)\right)
$$

where the LHS is the revenue loss of an increase in $\theta^{*}$ due to the nonparticipation of pivotal type and the RHS is the revenue gain from charging a higher price on all agents above the pivotal type $\theta^{*}$.

\subsection{Comparison with competition}

It is well known that with a continuum of types a competitive equilibrium may fail to exist. In fact Riley (2001) showed the general non-existence of the Rothschild-Stiglitz equilibrium. This existence problem can be circumvented by resorting to the reactive equilibrium concept introduced by Riley (1979) and developed further by Engers and Fernandez (1987). A reactive equilibrium is a set of offers such that there exists no profitable deviation by any firm given that other firms can optimally react to this deviation by offering new contracts. Engers and Fernandez (1987) provide general conditions, for which the Pareto-dominant full-separating zero-profit set of contracts is the unique reactive equilibrium outcome. It turns out that those conditions hold true in our framework. ${ }^{12}$ The key element is that firms are deterred to deviate from the full separating equilibrium by the belief that other firms will react to "skim the cream" and make such initial deviation unprofitable.

The Pareto-dominant fully separating zero-profit competitive equilibrium solves

$$
\max _{\substack{\pi(.) \geq 0 \\ \delta(.) \in[0,1]}} S(\pi(\theta), \delta(\theta) ; \theta) \quad \forall \theta=[\underline{\theta}, \bar{\theta}]
$$

\footnotetext{
${ }^{12}$ The conditions for existence and uniqueness of a reactive equilibrium in our model are: (1) a continuous probability distribution $F(\theta) ;(2)$ the profit function of insurance firms is continuous, bounded and non increasing in $\theta$ and $\delta ;(3) V(\pi, \delta, \theta)$ is continuous on $\Theta \times \Delta \times \Pi$ where $\Delta=[0,1]$ and $\Pi=[\underline{\pi}, \bar{\pi}]$ with $\underline{\pi}=\inf \{\tilde{\pi}(\delta ; \theta): \theta \in \Theta$, $\delta \in \Delta\}$ and $\bar{\pi}=\sup \{\tilde{\pi}(\delta ; \theta): \theta \in \Theta, \delta \in \Delta\}$, is stricly decreasing in $\pi$ and satisfies the Single-Crossing property; (4) the contract space is a closed set $\Delta \times \Pi$.
} 
subject to

$$
\begin{aligned}
V(\pi(\theta), \delta(\theta) ; \theta) & \geq V(0,0 ; \theta) & & \forall \theta \in[\underline{\theta}, \bar{\theta}] \\
V(\pi(\theta), \delta(\theta) ; \theta) & \geq V(\pi(\hat{\theta}), \delta(\hat{\theta}) ; \theta) & & \forall \theta, \hat{\theta} \in[\underline{\theta}, \bar{\theta}] \\
\pi(\theta)-\delta(\theta) \theta & =0 & & \forall \theta \in[\underline{\theta}, \bar{\theta}]
\end{aligned}
$$

Following Hindriks and De Donder (2003) the solution involves the following coverage function

$$
\delta^{c}(\theta)=(\theta / \bar{\theta})^{\frac{1}{\alpha}} \in[0,1]
$$

So, while in the monopoly everyone above $\theta^{*}$ gets full insurance, with competition only the highest-risk individuals obtain full coverage and all the other individuals with lower risk obtain partial coverage. On the other hand everyone below $\theta^{*}$ gets no insurance with monopoly, while they are provided at least with partial coverage in the competitive case. Figure 3 compares equilibrium coverage with monopoly and competition.

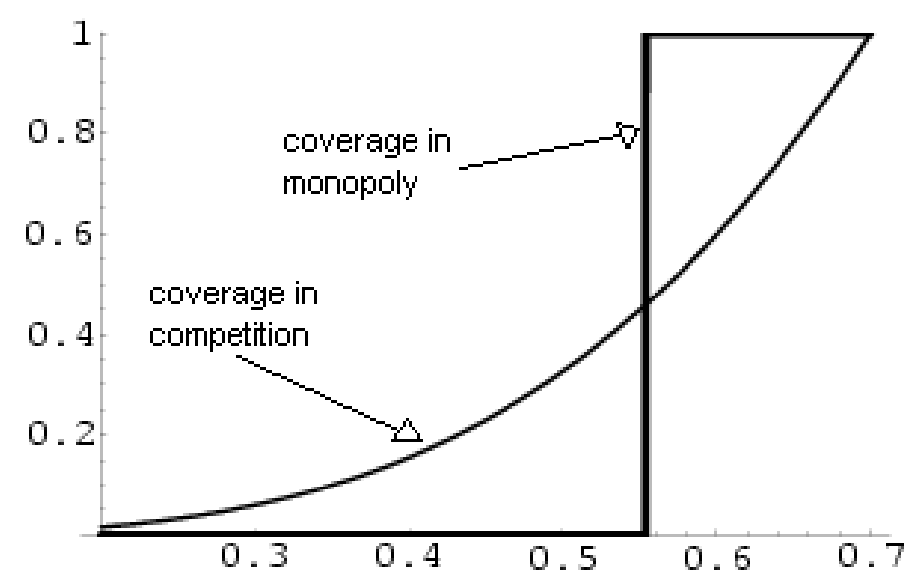

Figure 3: Relative coverage rates under competition and monopoly.

An interesting difference is that the distribution of risk does not influence the screening equilibrium in the competitive setting, while it influences the equilibrium allocation in a monopoly; this feature, as we will see shortly, is essential in the comparison of the outcomes.

Turning to competitive prices, each type is charged the fair premium

$$
\pi^{c}(\theta)=\theta(\theta / \bar{\theta})^{\frac{1}{\alpha}}
$$

The payoff of type $\theta$ under competition is:

$$
\begin{aligned}
V^{c}(\theta) & =\omega-\pi^{c}(\theta)-(1+\alpha)\left(1-\delta^{c}(\theta)\right) \theta \\
& =\omega-(1+\alpha) \theta+\alpha \theta(\theta / \bar{\theta})^{\frac{1}{\alpha}}
\end{aligned}
$$


The payoff of type $\theta$ under monopoly is:

$$
V^{m}(\theta)=\omega-(1+\alpha) \min \left\{\theta, \theta^{*}\right\}
$$

Define the payoff difference between competition and monopoly equilibrium:

$$
\Delta V(\theta)=V^{c}(\theta)-V^{m}(\theta)
$$

Hence

$$
\begin{aligned}
\Delta V(\theta) & =\alpha \theta(\theta / \bar{\theta})^{\frac{1}{\alpha}} \geq 0 & & \forall \theta<\theta^{*} \\
& =\alpha \theta(\theta / \bar{\theta})^{\frac{1}{\alpha}}-(1+\alpha)\left(\theta-\theta^{*}\right) & & \forall \theta \geq \theta^{*}
\end{aligned}
$$

so when $\theta \geq \theta^{*}$ it is not obvious that individuals prefer competition to monopoly. To see if individuals on average can be better off with monopoly, we integrate the payoff difference over the whole range of types to obtain the following expression (see Appendix):

$$
\begin{aligned}
\int_{\underline{\theta}}^{\bar{\theta}} \Delta V(\theta) d F(\theta)=\int_{\underline{\theta}}^{\bar{\theta}} \alpha \theta(\theta / \bar{\theta})^{\frac{1}{\alpha}} d F(\theta) & (1+\alpha)\left(\bar{\theta}-\theta^{*}\right) \\
& +\frac{1}{2}(1+\alpha)\left[1-F^{2}\left(\theta^{*}\right)\right]
\end{aligned}
$$

Including the monopolist profit, we can compare the outcomes under competition and monopoly in terms of total surplus (see Appendix):

$$
\Delta W=\alpha\left\{\int_{\underline{\theta}}^{\theta^{*}} \theta(\theta / \bar{\theta})^{\frac{1}{\alpha}} d F(\theta)-\int_{\theta^{*}}^{\bar{\theta}} \theta\left[1-(\theta / \bar{\theta})^{\frac{1}{\alpha}}\right] d F(\theta)\right\}
$$

where the first part measures the benefit of competition in terms of better insurance to those who cannot buy insurance in a monopoly $\left(\theta<\theta^{*}\right)$; and the second part measures the cost of competition in providing only partial insurance to those who can get full insurance in a monopoly $\left(\theta \geq \theta^{*}\right)$. The net balance of the two effects depends on the size of the threshold $\theta^{*}$.

We can easily derive $\theta^{*}$ for a uniform distribution.

$$
f(\theta)=\frac{1}{\bar{\theta}-\underline{\theta}} ; \quad F(\theta)=\frac{\theta-\underline{\theta}}{\bar{\theta}-\underline{\theta}} ; \quad h(\theta)=\frac{1}{\bar{\theta}-\theta}
$$

by definition $\theta=\theta^{*}$ solves

$$
\begin{aligned}
\theta & =\frac{1+\alpha}{\alpha} \frac{1}{h(\theta)} \\
& =\frac{1+\alpha}{\alpha}(\bar{\theta}-\theta)
\end{aligned}
$$


Hence

$$
\theta^{*}=\frac{1+\alpha}{1+2 \alpha} \bar{\theta}
$$

In Figure 4 the effect of a change in distribution is depicted: the left panel shows a negatively skewed Beta distribution, while the right panel shows a positively skewed Beta distribution. While the coverage rate under competition is the same, the level of $\theta^{*}$ in the left panel is lower than in the right panel.
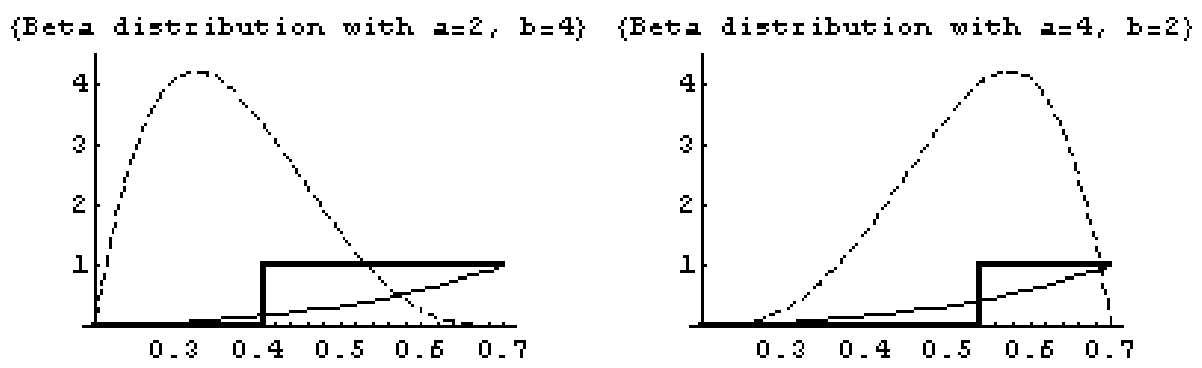

Figure 4: The effect of different distributions on coverage.

\subsection{Numerical simulation}

In this section we perform some numerical simulations using Beta distribution of risks with non-decreasing hazard rate.

We have seen that the risk distribution affects the monopoly outcome by changing the critical level $\theta^{*}$, while it does not affect the competition equilibrium outcome.

\begin{tabular}{|c||c|c|c|c|c|c|}
\hline \multicolumn{1}{|c||}{} & \multicolumn{7}{c|}{$\mathrm{b}$} \\
\hline $\mathrm{a}$ & 1 & 2 & 3 & 5 & 10 & 25 \\
\hline \hline 1 & 14.58 & 7.25 & 5.27 & 4.89 & 16.15 & 100 \\
2 & 25.35 & 15.23 & 11.76 & 10.22 & 16.94 & 70.19 \\
3 & 33.65 & 22.57 & 18.15 & 15.50 & 19.92 & 56.65 \\
5 & 45.64 & 34.62 & 29.37 & 25.14 & 26.24 & 48.89 \\
10 & 62.42 & 53.63 & 48.53 & 43.06 & 39.73 & 48.83 \\
25 & 80.36 & 75.68 & 72.48 & 68.15 & 62.75 & 60.64 \\
\hline
\end{tabular}

Table 1: Coverage rate under monopoly for various distributions over risk $\operatorname{Bet} a(a, b)$, given the parameters $\alpha=0.1, \underline{\theta}=0.3, \bar{\theta}=0.7$.

The effect of changing the distribution on the equilibrium monopoly coverage rate is illustrated in Table 1 . In this table a Beta distribution 
$(a, b)$ is used to show that the coverage rate increases with the concentration of the distribution (i.e., simultaneous increase in $a$ and $b$ ). Moreover, the more positively skewed is the distribution (i.e., higher $a-b>0$ ) the higher is the coverage rate.

The relative performance of competition and monopoly is shown in the following tables. They show the total surplus realized in the competitive and monopoly equilibria as a percentage of the total surplus under full information. Fixing the degree of risk aversion and the spread of risks we can compare competition and monopoly for different Beta distributions. The key result is that except for the uniform distribution $(a=b=1)$ and distributions for which the highest risk is the mode $(b=1)$, the monopoly realizes a higher fraction of the gains from trade.

Comparing Tables 2 and 3 illustrates the effect of an increase in risk aversion. The surplus increases for both competition and monopoly (due to larger demand for insurance), but the monopoly still significantly outperforms competition.

Comparing Table 3 and 4 we see the effect of a reduction in the spread of risks $[\underline{\theta}, \bar{\theta}]$. The effect is once again an increase in the surplus of both competition and monopoly without changing the relative performance.

\begin{tabular}{|c||cc|cc|cc|cc|cc|}
\hline \multicolumn{1}{|c||}{} & \multicolumn{10}{c|}{ b } \\
\cline { 2 - 11 } & \multicolumn{2}{|c|}{1} & \multicolumn{2}{c|}{2} & \multicolumn{2}{c|}{3} & \multicolumn{2}{|c|}{5} & \multicolumn{2}{c|}{10} \\
\hline \hline $\mathrm{a}$ & mon & comp & mon & comp & mon & comp & mon & comp & mon & comp \\
1 & 19.57 & 20.42 & 10.51 & 6.34 & 7.75 & 2.57 & 6.91 & 0.71 & 19.06 & 0.14 \\
2 & 30.13 & 31.18 & 19.37 & 12.37 & 15.34 & 5.83 & 13.31 & 1.85 & 20.18 & 0.35 \\
3 & 37.89 & 39.02 & 26.83 & 17.94 & 22.14 & 9.34 & 19.12 & 3.32 & 23.43 & 0.66 \\
5 & 48.93 & 50.09 & 38.46 & 27.56 & 33.32 & 16.36 & 29.03 & 6.88 & 29.94 & 1.56 \\
10 & 64.34 & 65.45 & 56.21 & 44.51 & 51.47 & 31.28 & 46.37 & 16.86 & 43.10 & 4.96 \\
\hline
\end{tabular}

Table 2: Surplus under monopoly and competition as a percentage of the First Best surplus for various distributions over risk Beta $(a, b)$, given the parameters $\alpha=0.1, \underline{\theta}=0.3, \bar{\theta}=0.7$. 


\begin{tabular}{|c||cc|cc|cc|cc|cc|}
\hline \multicolumn{1}{|c||}{} & \multicolumn{10}{c|}{ b } \\
\cline { 2 - 11 } & \multicolumn{2}{|c|}{1} & \multicolumn{2}{c|}{2} & \multicolumn{2}{c|}{3} & \multicolumn{2}{c|}{5} & \multicolumn{2}{c|}{10} \\
\hline \hline $\mathrm{a}$ & mon & comp & mon & comp & mon & comp & mon & comp & mon & comp \\
1 & 44.10 & 48.29 & 42.75 & 31.14 & 50.10 & 23.40 & 89.06 & 16.69 & 100 & 11.81 \\
2 & 57.77 & 61.41 & 54.94 & 43.52 & 56.81 & 33.76 & 65.22 & 23.90 & 84.60 & 15.55 \\
3 & 65.50 & 68.86 & 62.31 & 51.83 & 62.60 & 41.49 & 66.64 & 29.97 & 78.82 & 19.07 \\
5 & 74.37 & 77.35 & 71.25 & 62.61 & 70.45 & 52.44 & 71.38 & 39.64 & 77.23 & 25.46 \\
10 & 84.09 & 86.43 & 81.69 & 75.74 & 80.49 & 67.52 & 79.66 & 55.39 & 80.54 & 38.15 \\
\hline
\end{tabular}

Table 3: Surplus under monopoly and competition as a percentage of the First Best surplus for various distributions over risk Beta $(a, b)$, given the parameters $\alpha=0 . \overline{3}, \underline{\theta}=0.3, \bar{\theta}=0.7$.

\begin{tabular}{|c||cc|cc|cc|cc|cc|}
\hline \multicolumn{1}{|c||}{} & \multicolumn{10}{c|}{ b } \\
\cline { 2 - 11 } & \multicolumn{2}{|c|}{1} & \multicolumn{2}{c|}{2} & \multicolumn{2}{c|}{3} & \multicolumn{2}{c|}{5} & \multicolumn{2}{c|}{10} \\
\hline \hline $\mathrm{a}$ & mon & comp & mon & comp & mon & comp & mon & comp & mon & comp \\
1 & 56.25 & 57.64 & 73.65 & 43.56 & 100 & 37.00 & 100 & 30.99 & 100 & 26.21 \\
2 & 67.97 & 69.55 & 71.15 & 55.06 & 77.16 & 46.89 & 87.21 & 38.26 & 96.24 & 30.34 \\
3 & 74.28 & 75.99 & 74.90 & 62.45 & 77.63 & 53.95 & 83.52 & 44.10 & 92.11 & 34.05 \\
5 & 81.24 & 83.00 & 80.44 & 71.60 & 81.09 & 63.51 & 83.52 & 52.95 & 89.06 & 40.43 \\
10 & 88.57 & 90.10 & 87.40 & 82.24 & 87.02 & 75.87 & 87.16 & 66.24 & - & - \\
\hline
\end{tabular}

Table 4: Surplus under monopoly and competition as a percentage of the First Best surplus for various distributions over risk Beta $(a, b)$, given the parameters $\alpha=0 . \overline{3}, \underline{\theta}=0.3, \bar{\theta}=0.5$.

\section{Conclusions}

There is recent work on the (asymptotic) efficiency of competition based on the idea that asymmetry in agent's information is relatively unimportant in a large economy (obtained by a replication process) because any single agent has only a small amount of information not known by the other agents. This is the notion of informational smallness. As Gul and Postlewaite noted this result holds in private value information problems in which agents have private information that is of direct relevance only to themselves (i.e. the agents' utility functions depend only on their own type). This is obviously not the case for the insurance problem (and for the adverse selection problem in general). Indeed in our insurance problem with a continuum of types it might seem that each agent is informationally small and yet the market outcome is very 
far from the full information outcome. The reason is that each agent remains informationally large in this context.

Using the benchmark model of Rothschild and Stiglitz (1976), we contrast the competitive equilibrium outcome with the monopoly equilibrium outcome à la Stiglitz (1977) and we compare their relative efficiency. The main change is that we adopt the dual theory of risk so that the comparison comes out neatly. The dual theory has the property that utility is linear in income, and risk aversion is expressed entirely by a transformation of probabilities in which bad outcomes are given relatively higher weights and good outcomes are given relatively lower weights.

Our main finding is that competition is bad and that the monopoly outcome in general is more efficient than the competitive outcome (according to our expected efficiency criterion defined as the fraction of the total surplus that is realized by the market). The reason why monopoly performs better than competition is that the monopolists can exploit its market power to relax the incentive constrains. This is one of many examples of the interplay between market imperfections. The economy, in effect has to trade off between two different imperfections: imperfections of information or imperfections of competition, with no particular reason that these imperfections will be balanced optimally.

We expect our result about the inefficiency of competition in insurance markets with adverse selection to carry over on other markets with adverse selection like the capital market or the job market. We also plan to extend this analysis to screening in the higher education market.

There is a final remark about the use of the dual theory of risk. With this specification there is no income effect on the demand of insurance. In contrast, the expected utility approach will raise the demand for insurance in the monopoly market relative to the competitive market if the absolute risk aversion is decreasing. This is because monopoly price is higher than competitive price which reduces income and thus raises the marginal willingness to pay for insurance. It is then expected that moving to the expect utility will further increase the amount of insurance in the monopoly market relative to the competitive market, thereby reinforcing our conclusion about the inefficiency of competition.

\section{Appendix}

\section{Proof of Proposition 1.}

The optimization program is a linear programme with 3 variables and

7 constraints (when accounting for the 3 nonnegativity constraints). In 
this problem there cannot be a solution with variables equal to zero. ${ }^{13}$ So excluding the nonnegativity constraints, the search of the solution amounts to the search of the extreme points among the remaining 4 constraints. From linear programming we know that when the nonnegativity constraints are not binding any basic feasible solution implies that the number of binding constraints must be equal to the total number of variables. ${ }^{14}$ So we must have a solution with three binding constraints. There are only two possibilities. Either $\delta(\underline{\theta})<1$ and we have a separating outcome or $\delta(\underline{\theta})=1$ and we have a pooling outcome.

Define $p^{*}=\frac{\bar{\theta}-\underline{\theta}-\alpha \underline{\theta}}{\bar{\theta}-\underline{\theta}}<1$.

When $p<p^{*}$, we have a separating outcome with $\delta(\underline{\theta})<1$.

In this case the constraints (9), (10) and (11) are binding. By (10)

$$
\pi(\underline{\theta})=(1+\alpha) \underline{\theta} \delta(\underline{\theta})
$$

and by (11)

$$
\pi(\bar{\theta})=\pi(\underline{\theta})+(1+\alpha)(1-\delta(\underline{\theta})) \bar{\theta}
$$

Substituting in (9) the solution is

$$
\begin{aligned}
\delta(\underline{\theta}) & =\frac{(1-p) \alpha \bar{\theta}}{(1-p)(1+\alpha)(\bar{\theta}-\underline{\theta})-p \alpha \underline{\theta}} \\
\delta(\bar{\theta}) & =1 \\
\pi(\underline{\theta}) & =\frac{(1-p)(1+\alpha) \alpha \bar{\theta} \underline{\theta}}{(1-p)(1+\alpha)(\bar{\theta}-\underline{\theta})-p \alpha \underline{\theta}} \\
\pi(\bar{\theta}) & =(1+\alpha) \bar{\theta}\left[\frac{(1-p)(\bar{\theta}-\underline{\theta})-p \alpha \underline{\theta}}{(1-p)(1+\alpha)(\bar{\theta}-\underline{\theta})-p \alpha \underline{\theta}}\right]
\end{aligned}
$$

where $\delta(\underline{\theta})<1$ for $p<p^{*}$.

Total welfare in the separating case is:

$$
(1-p) \alpha \bar{\theta}\left[\frac{(1-p)(\bar{\theta}-\underline{\theta})}{(1-p)(1+\alpha)(\bar{\theta}-\underline{\theta})-p \alpha \underline{\theta}}\right]
$$

When $p \geq p^{*}$ we have a pooling outcome with $\delta(\underline{\theta})=1$.

\footnotetext{
${ }^{13} \pi(\bar{\theta})$ cannot be zero because otherwise (9) would be violated. If $\delta(\underline{\theta})$ was equal to zero, so should be $\pi(\underline{\theta})$ for the constraint (10). Moreover either (9) or (11) should be not binding: so it were always possible to increase $\delta(\underline{\theta})$ and to change $\pi(\underline{\theta})$ and $\pi(\bar{\theta})$ so that the constraints are respected and total welfare is increased. For example, if (11) is binding (9) is not and it is possible to reduce $\pi(\bar{\theta})$ increasing $\delta(\underline{\theta})$ and $\pi(\underline{\theta})$ without violating the constraints. If (9) is binding (11) is not and it is possible to increase $\delta(\underline{\theta})$ with a fair premium $\pi(\underline{\theta})$ without violating the constraints.

${ }^{14}$ See Chiang (1984).
} 
- Either (10) and (11) are binding, and the solution is :

$$
\begin{aligned}
\delta(\underline{\theta}) & =\delta(\bar{\theta})=1 \\
\pi(\underline{\theta}) & =\pi(\bar{\theta})=(1+\alpha) \underline{\theta}
\end{aligned}
$$

where $\Pi \geq 0$ for $p \geq p^{*}$;

- or (9) and (11) are binding, and the solution is

$$
\begin{aligned}
\delta(\underline{\theta}) & =\delta(\bar{\theta})=1 \\
\pi(\underline{\theta}) & =\pi(\bar{\theta})=(1-p) \bar{\theta}+p \underline{\theta}
\end{aligned}
$$

Moreover, any convex combination of the two pooling solutions is also a solution, differing only in the premium charged and the division of the surplus. So any premium $(1-p) \bar{\theta}+p \underline{\theta} \leq \pi(\underline{\theta})=\pi(\bar{\theta}) \leq(1+\alpha) \underline{\theta}$ is also a solution. Total welfare in the case of pooling is

$$
p \alpha \underline{\theta}+(1-p) \alpha \bar{\theta}
$$

which completes the proof.

Proof of Proposition 2.

Substituting the binding constraints $(5),(8)$ and setting $\delta(\bar{\theta})=1$ the monopoly optimization problem reduces to

$$
\max _{\delta(\underline{\theta}) \in[0,1]}(1-p) \alpha \bar{\theta}+[p \alpha \underline{\theta}-(1-p)(1+\alpha)(\bar{\theta}-\underline{\theta})] \delta(\underline{\theta})
$$

The solution is

$$
\begin{array}{lll}
\delta(\underline{\theta})=1 & \text { if } & p \geq \frac{(1+\alpha)(\bar{\theta}-\underline{\theta})}{(1+\alpha)(\bar{\theta}-\underline{\theta})+\alpha \underline{\theta}} \equiv p^{\circ} \\
\delta(\underline{\theta})=0 & \text { if } & p<p^{\circ}
\end{array}
$$

In the case of pooling, by the binding constraint (5), the premium is $\pi(\underline{\theta})=\pi(\bar{\theta})=(1+\alpha) \underline{\theta}$. In the case of separating outcome, only the high type gets full insurance $\delta(\bar{\theta})=1$ at its reservation price $\pi(\bar{\theta})=$ $(1+\alpha) \bar{\theta}$. It is then easily checked that $p^{\circ}>p^{*}$ which completes the proof. $\square$

Proof of Proposition 4.

Because the unique binding IR constraint is for the lowest type:

$$
V(\pi(\underline{\theta}), \delta(\underline{\theta}) ; \underline{\theta})=V(0,0 ; \underline{\theta})
$$

The set of incentive constraints implies that

$$
\theta \in \arg \max _{\hat{\theta} \in[\underline{\theta}, \bar{\theta}]} V(\pi(\hat{\theta}), \delta(\hat{\theta}) ; \theta) \quad \forall \theta, \hat{\theta} \in[\underline{\theta}, \bar{\theta}]
$$


the first order condition for the type $\theta$ is:

$$
\begin{aligned}
\frac{\partial V(\pi(\hat{\theta}), \delta(\hat{\theta}) ; \theta)}{\partial \hat{\theta}} & =\frac{\partial}{\partial \hat{\theta}}[\omega-(1+\alpha)(1-\delta(\hat{\theta})) \theta-\pi(\hat{\theta})] \\
& =(1+\alpha) \delta^{\prime}(\hat{\theta}) \theta-\pi^{\prime}(\hat{\theta})=0
\end{aligned}
$$

which evaluated at $\hat{\theta}=\theta$ gives the local incentive compatibility conditions (LIC)

$$
(1+\alpha) \delta^{\prime}(\theta) \theta-\pi^{\prime}(\theta)=0 \quad \forall \theta \in[\underline{\theta}, \bar{\theta}]
$$

Moreover, the necessary LIC is also sufficient condition when the utility function $V(\pi(\hat{\theta}), \delta(\hat{\theta}) ; \theta)$ satisfies the increasing differences property,

$$
\frac{\partial^{2} V(\pi(\hat{\theta}), \delta(\hat{\theta}) ; \theta)}{\partial \hat{\theta} \partial \theta}=(1+\alpha) \delta^{\prime}(\hat{\theta}) \geq 0
$$

which requires the coverage to be monotonically increasing $\delta^{\prime}(\theta) \geq 0$. Define the value function of the maximization problem evaluated at the truth-telling equilibrium:

$$
U(\theta)=V(\pi(\theta), \delta(\theta) ; \theta)=\omega-(1+\alpha)(1-\delta(\theta)) \theta-\pi(\theta)
$$

differentiating w.r.t. $\theta$

$$
\begin{aligned}
U^{\prime}(\theta) & =-(1+\alpha)(1-\delta(\theta))+(1+\alpha) \delta^{\prime}(\theta) \theta-\pi^{\prime}(\theta) \\
& =-(1+\alpha)(1-\delta(\theta))<0
\end{aligned}
$$

where the second equality follows from (19).

Using these results we can rewrite the maximization programme of the monopolist as follows:

$$
\max _{\pi(\theta), \delta(\theta)} \int_{\underline{\theta}}^{\bar{\theta}}[\pi(\theta)-\delta(\theta) \theta] d F(\theta)
$$

subject to

$$
\begin{aligned}
\delta^{\prime}(\theta) & \geq 0 \\
(1+\alpha) \delta^{\prime}(\theta) \theta-\pi^{\prime}(\theta) & =0 \\
V(\pi(\underline{\theta}), \delta(\underline{\theta}) ; \underline{\theta}) & \geq V(0,0 ; \underline{\theta})
\end{aligned}
$$

Ignoring for the moment the monotonicity constraint that will be checked later, we can rewrite the objective function after substituting the constraint in it. From the definition of the value function

$$
\pi(\theta)=\omega-(1+\alpha)(1-\delta(\theta)) \theta-U(\theta)
$$


and

$$
\begin{aligned}
U(\theta) & =U(\underline{\theta})+\int_{\underline{\theta}}^{\theta}-(1+\alpha)(1-\delta(s)) d s \\
& =\omega-(1+\alpha) \underline{\theta}-(1+\alpha)(\theta-\underline{\theta})+\int_{\underline{\theta}}^{\theta}(1+\alpha) \delta(s) d s \\
& =\omega-(1+\alpha) \theta+\int_{\underline{\theta}}^{\theta}(1+\alpha) \delta(s) d s
\end{aligned}
$$

Substituting this expression into (20):

$$
\begin{aligned}
\pi(\theta) & =\omega-(1+\alpha)(1-\delta(\theta)) \theta-\omega+(1+\alpha) \theta-\int_{\underline{\theta}}^{\theta}(1+\alpha) \delta(s) d s \\
& =(1+\alpha) \delta(\theta) \theta-\int_{\underline{\theta}}^{\theta}(1+\alpha) \delta(s) d s
\end{aligned}
$$

This expression for the insurance premium captures the incentive and participation constraints. Plugging this premium in the objective function we get the reduced problem:

$$
\max _{\delta(\theta)} \int_{\underline{\theta}}^{\bar{\theta}}\left[(1+\alpha) \delta(\theta) \theta-\delta(\theta) \theta-\int_{\underline{\theta}}^{\theta}(1+\alpha) \delta(s) d s\right] d F(\theta)
$$

The second term is the aggregate informational rent which integrating by parts is given by

$\int_{\underline{\theta}}^{\bar{\theta}} \int_{\underline{\theta}}^{\theta}(1+\alpha) \delta(s) d s d F(\theta)=\left|\int_{\underline{\theta}}^{\theta}(1+\alpha) \delta(s) d s F(\theta)\right|_{\underline{\theta}}^{\bar{\theta}}-\int_{\underline{\theta}}^{\bar{\theta}}(1+\alpha) \delta(\theta) F(\theta) d \theta$

with

$$
\begin{aligned}
\int_{\underline{\theta}}^{\underline{\theta}}(1+\alpha) \delta(s) d s & =0 \\
F(\underline{\theta}) & =0 \\
F(\bar{\theta}) & =1
\end{aligned}
$$

Hence:

$$
\begin{aligned}
\int_{\underline{\theta}}^{\bar{\theta}} \int_{\underline{\theta}}^{\theta}(1+\alpha) \delta(s) d s d F(\theta) & =\int_{\underline{\theta}}^{\bar{\theta}}(1+\alpha) \delta(\theta) d \theta-\int_{\underline{\theta}}^{\bar{\theta}}(1+\alpha) \delta(\theta) F(\theta) d \theta \\
& =\int_{\underline{\theta}}^{\bar{\theta}} \frac{1-F(\theta)}{f(\theta)}(1+\alpha) \delta(\theta) d F(\theta)
\end{aligned}
$$


Plugging the solution for the informational rent into the objective function

$$
\max _{\delta(\theta) \in[0,1]} \int_{\underline{\theta}}^{\bar{\theta}}\left[\alpha \delta(\theta) \theta-\frac{1-F(\theta)}{f(\theta)}(1+\alpha) \delta(\theta)\right] d F(\theta)
$$

Let $h(\theta)=\frac{f(\theta)}{1-F(\theta)}$ be the hazard rate, then the monopoly programme is

$$
\max _{\delta(\theta) \in[0,1]} \int_{\underline{\theta}}^{\bar{\theta}}\left[\alpha \delta(\theta) \theta-\frac{(1+\alpha)}{h(\theta)} \delta(\theta)\right] d F(\theta)
$$

Because the objective is maximized when the argument of the integral is maximized $\forall \theta \in[\underline{\theta}, \bar{\theta}]$ the result of Proposition 4 is obtained.

It remains to check the monotonicity constraint. To be verified, it requires that

$$
\frac{\partial^{2}}{\partial \delta(\theta) \partial \theta}\left[\alpha \delta(\theta) \theta-\frac{(1+\alpha)}{h(\theta)} \delta(\theta)\right] \geq 0
$$

This condition can be expressed in terms of the hazard rate

$$
\frac{h^{\prime}(\theta)}{h^{2}(\theta)} \geq-\frac{\alpha}{1+\alpha}
$$

A sufficient condition is that the hazard rate is non-decreasing in the interval $[\underline{\theta}, \bar{\theta}]$ which completes the proof. $\square$

Welfare comparison in the continuum case.

Integrating the payoff difference over the whole range of types

$$
\begin{aligned}
\int_{\underline{\theta}}^{\bar{\theta}} \Delta V(\theta) d F(\theta)=\int_{\underline{\theta}}^{\bar{\theta}}\left\{\alpha \theta(\theta / \bar{\theta})^{\frac{1}{\alpha}}-(1+\alpha)\left[\theta-\min \left(\theta, \theta^{*}\right)\right]\right\} d F(\theta) \\
=\int_{\underline{\theta}}^{\bar{\theta}} \alpha \theta(\theta / \bar{\theta})^{\frac{1}{\alpha}} d F(\theta)-\int_{\underline{\theta}}^{\bar{\theta}}(1+\alpha) \theta d F(\theta) \\
\quad+\int_{\underline{\theta}}^{\theta^{*}}(1+\alpha) \theta d F(\theta)+\int_{\theta^{*}}^{\bar{\theta}}(1+\alpha) \theta^{*} d F(\theta) \\
=\int_{\underline{\theta}}^{\bar{\theta}} \alpha \theta(\theta / \bar{\theta})^{\frac{1}{\alpha}} d F(\theta)-\int_{\theta^{*}}^{\bar{\theta}}(1+\alpha) \theta d F(\theta) \\
+\int_{\theta^{*}}^{\bar{\theta}}(1+\alpha) \theta^{*} d F(\theta)
\end{aligned}
$$


where

$$
\begin{aligned}
\int_{\theta^{*}}^{\bar{\theta}}(1+\alpha) \theta^{*} d F(\theta) & =(1+\alpha) \theta^{*}\left[1-F\left(\theta^{*}\right)\right] \\
\int_{\theta^{*}}^{\bar{\theta}}(1+\alpha) \theta d F(\theta) & =(1+\alpha)[\theta F(\theta)]_{\theta^{*}}^{\bar{\theta}}-(1+\alpha) \int_{\theta^{*}}^{\bar{\theta}} F(\theta) d \theta \\
& =(1+\alpha) \bar{\theta}-(1+\alpha) \theta^{*} F\left(\theta^{*}\right)-\frac{1}{2}(1+\alpha)\left[1-F^{2}\left(\theta^{*}\right)\right]
\end{aligned}
$$

which reduces to

$$
\begin{aligned}
\int_{\underline{\theta}}^{\bar{\theta}} \Delta V(\theta) d F(\theta)=\int_{\underline{\theta}}^{\bar{\theta}} \alpha \theta(\theta / \bar{\theta})^{\frac{1}{\alpha}} d F(\theta) & \\
& -(1+\alpha)\left(\bar{\theta}-\theta^{*}\right)+\frac{1}{2}(1+\alpha)\left[1-F^{2}\left(\theta^{*}\right)\right]
\end{aligned}
$$

In order to carry out the efficiency comparison we define the aggregate difference in consumer and producer surplus in the competitive and monopoly cases :

$$
\begin{aligned}
\Delta W= & \int_{\underline{\theta}}^{\bar{\theta}} \Delta V(\theta) d F(\theta)-\int_{\theta^{*}}^{\bar{\theta}}\left((1+\alpha) \theta^{*}-\theta\right) d F(\theta) \\
= & \int_{\underline{\theta}}^{\bar{\theta}} \alpha \theta(\theta / \bar{\theta})^{\frac{1}{\alpha}} d F(\theta)-\int_{\theta^{*}}^{\bar{\theta}}(1+\alpha) \theta d F(\theta) \\
& \quad+\int_{\theta^{*}}^{\bar{\theta}}(1+\alpha) \theta^{*} d F(\theta)-\int_{\theta^{*}}^{\bar{\theta}}(1+\alpha) \theta^{*} d F(\theta)+\int_{\theta^{*}}^{\bar{\theta}} \theta d F(\theta) \\
= & \int_{\underline{\theta}}^{\bar{\theta}} \alpha \theta(\theta / \bar{\theta})^{\frac{1}{\alpha}} d F(\theta)-\int_{\theta^{*}}^{\bar{\theta}} \alpha \theta d F(\theta) \\
= & \alpha\left\{\int_{\underline{\theta}}^{\theta^{*}} \theta(\theta / \bar{\theta})^{\frac{1}{\alpha}} d F(\theta)-\int_{\theta^{*}}^{\bar{\theta}} \theta\left[1-(\theta / \bar{\theta})^{\frac{1}{\alpha}}\right] d F(\theta)\right\}
\end{aligned}
$$

\section{References}

[1] Chassagnon, A. and B. Villeneuve, (2005), "Optimal risk-sharing under adverse selection and imperfect risk perception", Canadian Journal of Economics, 39, forthcoming.

[2] Chiang, A. C., (1984), Fundamental Methods of Mathematical Economics, McGraw-Hills, Singapore.

[3] Cho, I.-K. and D.M. Kreps, (1987), "Signalling games and stable equilibria", Quarterly Journal of Economics, 102, 179-221.

[4] Cohen, M. D., (1995), "Risk aversion concepts in expected- and non-expected-utility models", The Geneva Papers on Risk and Insurance Theory, 20, 73-91. 
[5] Crocker, K. J. and A. Snow, (1985), "The efficiency of competitive equilibria in insurance markets with asymmetric information", Journal of Public Economics, 26, 207-219.

[6] Dahlby, B., (1987), "Monopoly versus competition in an insurance market with adverse selection", The Journal of Risk and Insurance, 54 (2), 325-331.

[7] De Donder P. and J. Hindriks (2003), "The politics of redistributive social insurance", Journal of Public Economics, 87, 2639-2660.

[8] Doherty, N. A. and L. Eeckhoudt, (1995), "Optimal insurance without expected utility: The dual theory and the linearity of insurance contracts," Journal of Risk and Uncertainty, 10, 157-179.

[9] Engers, M. and L. Fernandez, (1987), "Market equilibrium with hidden knowledge and self-selection", Econometrica, 55, 425-39.

[10] Gresik, T and M.A. Satterthwaite (1989) "The rate at which a simple market becomes efficient as the number of traders increases: an asymptotic result for optimal trading mechanisms", Journal of Economic Theory, 48, 304-32.

[11] Gul, F. and A. Postlewaite (1992) "Asymptotic efficiency in large exchange economies with asymmetric information" Econometrica, 60, 1273-92.

[12] Harris, M. and R.M. Townsend, (1981) "Resource allocation under asymmetric information," Econometrica,. 49 (1), 33-64.

[13] Jaffe, D. and J.E Stiglitz (1990), "Credit rationing" in B. Friedman and F. Hahn, eds., Handbook of Monetary Economics, Vol. 2, Amsterdam: North-Holland, 837-88.

[14] Jeleva, M. and B. Villeneuve, (2004), "Insurance contracts with imprecise probabilities and adverse selection", Economic Theory, $23,777-794$.

[15] Laffont, J.-J and J. Tirole, (1993), A theory of incentives in procurement regulation, MIT Press, Cambridge, Massachusetts.

[16] Machina, M. J., (1989), "Dynamic consistency and non-expected utility models of choice under uncertainty", Journal of Economic Literature, 27 (4), 1622-1668.

[17] Machina, M. J., (1995), "Non-expected utility and the robustness of the classical insurance paradigm", in: Gollier, Christian and Machina, Mark J. (Eds.), Non expected utility and risk management, Kluwer Academic, Boston.

[18] Mailath, G. J., (1987), "Incentive compatibility in signaling games with continuum of types", Econometrica, 55 (6), 1349-1365.

[19] Maskin, E. and J. Riley, (1984), "Monopoly with incomplete information", Rand Journal of Economics, 15 (2), 171-196.

[20] Miyazaki, H., (1977), "The rat race and internal labor markets", 
Bell Journal of Economics, 8, 394-418.

[21] Mossin, J., (1968), "Aspects of rational insurance purchasing", Journal of Political Economy, 76, 533-568.

[22] Muthoo, A. and S. Mutuswami, (2005), "Competition and efficiency in markets with quality uncertainty", University of Essex Department of Economics Discussion Paper, 593.

[23] Riley, J. G., (1975), "Competitive signalling", Journal of Economic Theory, 10 (2), 174-186.

[24] Riley, J. G., (1985), "Competition with hidden knowledge", Journal of Political Economy, 93, 958-976.

[25] Riley, J. G., (2001), "Silver signals: twenty-five years of screening and signalling", Journal of Economic Literature, 39 (2), 432-478.

[26] Rustichini, A., M.A. Satterthwaite and S.R. Williams (1994), "Convergence to efficiency in a simple market with incomplete information" Econometrica, 62, 1041-64.

[27] Rothschild, M. and J.E. Stiglitz, (1976), "Equilibrium in competitive insurance markets: An essay on the economics of imperfect information", Quarterly Journal of Economics, 90 (4), 629-649.

[28] Satterthwaite, M.A., and S.R. Williams (1989), "The rate of convergence to efficiency in the buyer's bid double auction as the market becomes large", Review of Economic Studies, 56, 477-98.

[29] Segal, U. and A. Spivak, (1990), "First order versus second order risk aversion", Journal of Economic Theory, 51 (1), 111-125.

[30] Spence, M., (1978), "Product differentiation and performance in insurance markets", Journal of Public Economics, 10, 427-447.

[31] Stiglitz, J. E., (1977), "Monopoly, non-linear pricing and imperfect information: the insurance market", Review of Economic Studies, 44, 407-430.

[32] Stiglitz, J.E., (1975), "Information and economic analysis", in J.M. Parlin and A.R. Nobay, eds, Current Economic Problems. Cambridge: Cambridge University Press, 27-52.

[33] Topkis, D. M., (1998), Submodularity and Complementarity, Princeton University Press, Princeton, New Jersey.

[34] Wilson, C., (1977), "A model of insurance markets with incomplete information", Journal of Economic Theory, 12, 167-207.

[35] Yaari, M., (1987), "The dual theory of choice under risk", Econometrica, 55 (1), 95-115. 
Département des Sciences Économiques de l'Université catholique de Louvain

Institut de Recherches Économiques et Sociales

Place Montesquieu, 3

1348 Louvain-la-Neuve, Belgique 